\title{
Quick Exposure Check (QEC): a cross- cultural adaptation into Brazilian-Portuguese
}

\author{
Maria Luiza C.Comper ${ }^{\mathrm{a}, \mathrm{b}^{*}}$, Leonardo O.P. Costa ${ }^{\mathrm{b}, \mathrm{c}}$ and Rosimeire S. Padula \\ ${ }^{a}$ União Metropolitana de Ensino e Cultura, Av J.S. Pinheiro, Itabuna, Brazil \\ ${ }^{b}$ Universidade Cidade de São Paulo, R. Cesário Galeno, 448, São Paulo, Brazil \\ ${ }^{c}$ Musculoskeletal Division, The George Institute for Global Health, Sydney, Australia
}

\begin{abstract}
Most instruments used by occupational safety and health professionals have been originally developed in English. Cross-cultural adaptation enables the use of these instruments in other countries, cultures and languages in countries like Brazil, where the official language is not English. This methodology ensures that the translation process includes the cultural and linguistic equivalence between the original instrument and the translated version. Therefore, this study aimed to translate and cross-culturally adapt the instrument Quick Exposure Check (QEC) into Brazilian-Portuguese. The process of cross-cultural adaptation followed the steps of translation, synthesis, back-translation, expert committee review and pretesting. The final questionnaire was pretested by two raters in 40 workers employed in an expedition of knitting and textile industry. All versions resulting from the translation process $(\mathrm{T} 1, \mathrm{~T} 2)$ and back-translation (BT1, BT2) showed good agreement. During the pre-test was not presented any difficulty in understanding or completing the instrument adapted QEC for Brazilian language.
\end{abstract}

Keywords: musculoskeletal disorders; ergonomic risk factors; occupational health

\section{Introduction}

A high occurrence of Musculoskeletal Disorders (MSDs) in workers in Brazil ${ }^{[2]}$ has motivated the development of strategies to reduce risk factors and improvements in working conditions. It is important to develop risk analysis aiming to identify occupational tasks which require ergonomic interventions. The best analysis would be observational protocols and questionnaires ${ }^{[9,4]}$.

However, most instruments used by occupational safety and health professionals have been originally developed in English. Thus, research in countries like Brazil where the official language is not English is hampered with regards to these instruments and whenever these instruments were used just literal translation was performed ${ }^{[3,6]}$. The best way would be by cross-culturally adapting these instruments, which will enable their use in other countries, cultures and languages. In addition to being economical, this procedure facilitates the comparison of results from the same questionnaire in different cultures ${ }^{[1]}$.
This study, therefore, aimed to translate and crossculturally adapt the Quick Exposure Check (QEC) instrument into Brazilian Portuguese.

\subsection{Quick Exposure Check (QEC)}

QEC is an instrument that evaluates occupational risk factors. It covers twelve topics from a total of sixteen questions, which are distributed in two columns. The first column contains the observer's evaluation of the postures adopted and the frequency of movements performed by the cervical spine (neck), lumbar spine, shoulders and arms, wrists and hands. The second column contains the worker's opinions about the amount of weight handled, the time necessary to complete a given task, the level of hand force exerted, visual demands, vibration, driving of vehicles, work pacing and stress. The total QEC score combines the responses of both the evaluator and the worker for the following domains: posture versus strength, duration versus strength, posture versus duration as well as other specific risk factors. The

*Corresponding author. E-mail: marialuizacaires21@hotmail.com 
total score ranges from 46 to 269 points and can be classified in four categories of risk exposure: low (46-84 points), moderate (106-138 points), high (168198 points) and very high (187-242 points $)^{[4,5]}$.

\section{Methods}

The process of translation and cross-cultural adaptation followed the current guidelines recommended for this type of study ${ }^{[1,7]}$.

\subsection{Translation into Brazilian-Portuguese language}

The questionnaire and instructions have been translated into Portuguese by two bilingual (Portuguese and English) translators whose native language was Brazilian-Portuguese. One of the translators $\left(T_{1}\right)$ had experience in health terminology and was familiar with occupational issues regarding task assessment. The other translator $\left(\mathrm{T}_{2}\right)$ had no experience neither in health care nor knowledge of occupational task assessment. Both translators produced independent translations $\left(\mathrm{T}_{1}\right.$ and $\left.T_{2}\right)$.

\subsection{Synthesis of the translations $\left(T_{1}+T_{2}\right)$}

The independent translated versions were compared and analyzed. A consensus approach was used to resolve any differences. This process resulted in one consensual translation of the questionnaire $\left(T_{12}\right)$.

\subsection{Back-translation into English}

This synthesized Brazilian-Portuguese version was back-translated into English by two additional independent translators fluent in Portuguese and English. The translators were not familiar with the concepts explored in the questionnaire and had no knowledge of the original English version of the questionnaire.

\subsection{Back-translation synthesis $\left(B T_{1}+B T_{2}\right)$}

This step resulted in a synthesized English version $\left(\mathrm{BT}_{12}\right)$. This version was evaluated and compared with the original version in order to identify possible discrepancies.

\subsection{Expert committee review}

All versions obtained by the translation and backtranslation procedures were evaluated by an expert committee. This committee was composed by six bilingual professionals specialized in the knowledge area related to the questionnaire. They included two physical therapists experienced in occupational health, two physical therapists specialized in cross-cultural adaptation, and two translators. In this step, the translations were compared and the semantic, idiomatic, experimental and conceptual frameworks of the Brazilian-Portuguese version were analyzed. Few changes were made in the final version during this stage.

\subsection{Pre-testing}

The final version of the questionnaire was pretested by two examiners in 41 workers from the knitting and shipping sectors of the textile industry. The participants completed the adapted questionnaire and were individually interviewed about their understanding of the questions and possible responses. The inclusion criterion for this step was a minimum of six months of experience at the plant.

\subsection{Data analysis}

The results were analyzed by descriptive statistics (frequencies, means, standard deviation). The statistical program SPSS (version 17.0) was used for all analyses.

\subsection{Ethics approval}

All subjects were informed about the objectives and procedures of the study and were invited to participate by signing an informed consent form that had been approved by the local ethics committee (number 1658/2010).

\section{Results}

\subsection{Translation and cross-cultural adaptation}

The versions resulting from the translation ( $\mathrm{T} 1$, T2) and back-translation (BT1, BT2) steps showed good agreement, requiring only simple grammatical changes such as syntactical inversions or replacing some terms with synonyms in order to facilitate un- 
derstanding. Appendix 1 presents the QEC translated and cross-culturally adapted into BrazilianPortuguese.

\subsection{Description of the sample}

A total of 41 workers participated in the study, $46.3 \%$ were female and $53.7 \%$ were men. The mean age of the participants was 27,8 years (SD 3,1). All participants had at least finished high school.

\subsection{Pre-testing questionnaire}

All workers and evaluators participating in the pre-test showed no difficulty in understanding the Brazilian-Portuguese version of the QEC. The evaluators reported that the options for response for spine and shoulder were not very objective for assessing biomechanical risks.

\section{Discussion}

Many instruments are available for assessing worker exposure to ergonomic risk factors ${ }^{[4]}$. There are 30 instruments (observational methods) for the assessment of posture, movements and frequency of material handling ${ }^{[10]}$. The instrument selected for this study, the Quick Exposure Check (QEC) is fast, simple to use and applicable to a wide range of work tasks ${ }^{[5]}$.

The process of translation and cross-cultural adaptation followed the guidelines recommended for this type of study following the steps of translation, synthesis, back translation, review by a committee of experts and pre-testing ${ }^{[1,7] \text {. }}$

The translation and cultural adaptation process did not require major adjustments or modifications in QEC original version. This may be related to the characteristics of the instrument, originally built with feedback from experienced professionals in occupational health ${ }^{[8]}$. The QEC instrument is easy to understand because it has a simple format, with small texts and easy-to-understand terms. This characteristic eases the use of this tool by professionals with different scope of knowledge.

The workers and evaluators had no difficulty in understanding the instrument during the pre-testing stage. However, the evaluators had doubts about the items related to the spine and shoulder biomechanical assessment. This result demonstrates that the choice of any instrument is highly dependent on the real work situation, which varies widely. In this case, the experience of the technical evaluator may have influenced the analysis of occupational risk.

The evaluation of the clinimetric properties of the version adapted cross-culturally for Brazil is being checked by the authors of this study. The reliability will be calculated using a test-retest design and the validity will be assessed by comparing the adapted version of the QEC with the Job Factors Questionnaire $^{[3]}$.

\section{Conclusion}

The adapted version of the QEC into BrazilianPortuguese proved to be easy to understand and practical. But it requires training and experience for decision-making in the assessment of ergonomic risks.

\section{References}

[1] Beaton DE, Bombardier C, Guillemin F, Ferraz MB. Guidelines for the process of cross-cultural adaptation of self-report measures. Spine (Phila Pa 1976). 2000;25(24):3186-91.

[2] Brasil. Anuário Estatístico da Previdência Social 2009. Available from: ttp://www.mpas.gov.br/conteudoDinamico.php?id=989.

[3] Coluci M, Alexandre N, Rosecrance J. Reliability and validity of an ergonomics-related Job Factors Questionnaire. International Journal of Industrial Ergonomics. 2009;39(6):995-1001.

[4] David GC. Ergonomic methods for assessing exposure to risk factors for work-related musculoskeletal disorders. Occup Med (Lond). 2005;55(3):190-9.

[5] David G, Woods V, Li G, Buckle P. The development of the Quick Exposure Check (QEC) for assessing exposure to risk factors for work-related musculoskeletal disorders. Appl Ergon. $2008 ; 39(1): 57-69$.

[6] Giusti E, Befi-Lopes D. Tradução e adaptação transcultural de instrumentos estrangeiros para o Português Brasileiro (PB). Pró-Fono Revista de Atualização Científica. 2008;20(3):20710.

[7] Guillemin F, Bombardier C, Beaton D. Cross-cultural adaptation of health-related quality of life measures: literature review and proposed guidelines. J Clin Epidemiol. 1993;46(12):141732.

[8] Li G, Buckle P. Evaluating Change in Exposure to Risk for Musculoskeletal Disorders-A Practical Tool. Sudbury, Suffolk: HSE Books; 1999.

[9] Stock SR, Fernandes R, Delisle A, Vezina N. Reproducibility and validity of workers' self-reports of physical work demands. Scand J Work Environ Health. 2005;31(6):409-37.

[10] Takala EP, Pehkonen I, Forsman M, Hansson GA, Mathiassen SE, Neumann WP, et al. Systematic evaluation of observational methods assessing biomechanical exposures at work. Scand J Work Environ Health. 2010;36(1):3-24. 


\section{Appendix 1}

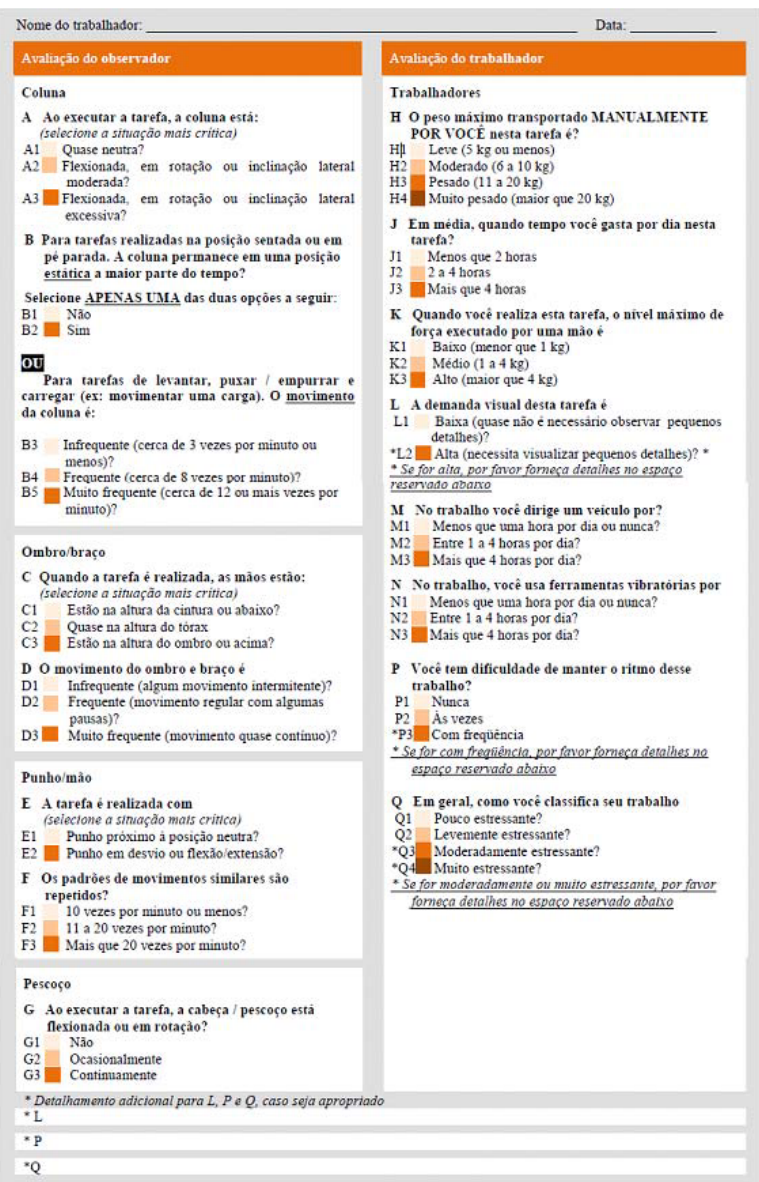

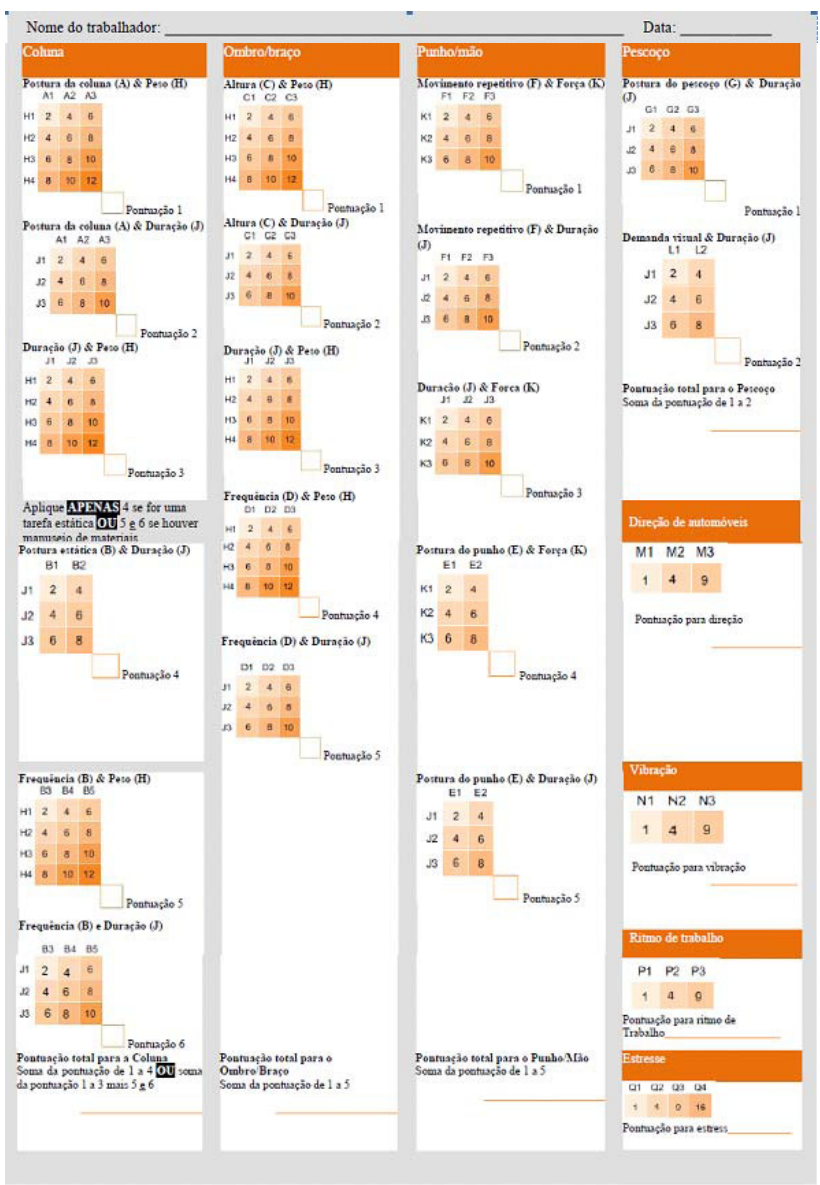

